\title{
Synthesis and Study of Antibacterial and Antifungal Activities of Novel 3-Alkyl-2,6-diarylpiperidin-4-one-2- thienoyl Hydrazone
}

\author{
M. MANIMARAN ${ }^{1}$, A. GANAPATHI ${ }^{2, *}$ and T. BALASANKAR ${ }^{2}$ \\ ${ }^{1}$ Department of Chemistry, Annamalai University, \\ Annamalainagar- 608002, Tamilnadu, India \\ ${ }^{2}$ Chemistry Section FEAT, Annamalai University, \\ Annamalainagar- 608002, Tamilnadu, India \\ drtbalasankar@rediff.com
}

Received 1 January 2014 / Accepted 23 January 2014

\begin{abstract}
In the present work a new series of 3-alkyl-2,6-diarylpiperidin-4-one-2-thienoyl hydrazones have synthesized by the reaction of 3-alkyl-2, 6-diarylpiperidin-4-one with 2-thiophenecarboxylic acid hydrazide and characterized by spectral (IR, ${ }^{1} \mathrm{H}$ NMR, ${ }^{13} \mathrm{C}$ NMR, HSQC, HMBC, NOESY and COSY) techniques. The hydrazones were screened for their in vitro antibacterial and antifungal activities against some selected micro organism. The results show that, compounds with halo-substituents at para position of the phenyl showed good antibacterial profile and antifungal against all tested organism.
\end{abstract}

Keywords: Hydrazones, Antibacterial, Antifungal

\section{Introduction}

Heterocyclic ring systems having piperidin-4-one nucleus have aroused great interest in the past and recent years due to their wide variety of biological properties such as antitumor ${ }^{1,2}$ anti-inflammatory ${ }^{3}$ central nervous system ${ }^{4-9}$ local anaesthetic ${ }^{10}$ anticancer $^{11}$ and antimicrobial activities ${ }^{12}$ and their derivative piperidines are also biologically important and act as neurokinin receptor antogonists ${ }^{13}$, analgesic and antihypertensive agents ${ }^{14}$. The importance of piperidin-4-one as intermediate in the synthesis of a variety of compounds of physiologically active has been reviewed by Prostakov Gaivoronskaya ${ }^{15}$.

Thiophenecarboxylic acid hydrazide have attracted considerable attention due to the biological activity of the 2-thiophene moiety which has been widely recognized and practically applied in several drugs ${ }^{16-18}$, herbicides ${ }^{19}$ and fungicides ${ }^{20,21}$. Thiophenecarboxylic hydrazide were tested for antituberculous activity ${ }^{22}$ and found to show $\beta$ adrenergic blocking activities ${ }^{23}$.

Synthesis of molecules, which are novel still resembling known biologically active molecules by virtue of the presence of some critical structural features, is an essential 
component of the search for new leads in drug designing programme. Certain small heterocyclic molecules act as highly functionalized scaffolds and are known pharmacophores of a number of biologically active and medicinally useful molecules. In the interest of above, we planned to synthesize a system, which combines both bio active piperidine and 2thiophenecarboxylic acid hydrazide components together to give a title compounds.

\section{Experimental}

All the reagents were commercially available and used without further purification. Solvents were distilled from appropriate drying agents immediately prior to use.

\section{Physical measurements}

The course of reaction and the purity were ascertained by performing TLC, melting points were determined in open capillaries and are uncorrected. IR spectra were recorded in PerkinElmer 297 spectrophotometer with $\mathrm{KBr}$ pellets. ${ }^{1} \mathrm{H}$ NMR spectra were recorded at $400 \mathrm{MHz}$ on Bruker AMX-400MHz spectrophotometer in $\mathrm{CDCl}_{3}$ using tetramethyl silane (TMS) as internal standard and ${ }^{13} \mathrm{C}$ NMR spectra were recorded at $100 \mathrm{MHz}$ on Bruker AMX$400 \mathrm{MHz}$ spectrophotometer in $\mathrm{CDCl}_{3}$. Elemental analyses $(\mathrm{C}, \mathrm{H}$ and $\mathrm{N})$ were carried out on a Carlo Erba model 1106 and Perkin Elmer models $240 \mathrm{CHN}$ analyzer. The results are within $\pm 0.4 \%$ of the theoretical values.

Table 1. Elemental analysis data

\begin{tabular}{cccccc}
\hline Compounds & $\mathrm{R}_{1}$ & $\mathrm{X}$ & $\begin{array}{c}\text { Carbon \% } \\
\text { (found /calculated) }\end{array}$ & $\begin{array}{c}\text { Hydrogen \% } \\
\text { (found /calculated) }\end{array}$ & $\begin{array}{c}\text { Nitrogen \% } \\
\text { (found /calculated) }\end{array}$ \\
\hline $\mathbf{1 4}$ & $\mathrm{CH}_{3}$ & $\mathrm{H}$ & $70.27 / 70.24$ & $5.67 / 5.71$ & $14.91 / 14.94$ \\
$\mathbf{1 5}$ & $\mathrm{CH}_{3}$ & $\mathrm{Cl}$ & $72.34 / 72.32$ & $6.15 / 6.14$ & $13.47 / 13.43$ \\
$\mathbf{1 6}$ & $\mathrm{CH}_{3}$ & $\mathrm{Br}$ & $71.25 / 71.24$ & $5.43 / 5.41$ & $12.36 / 12.35$ \\
$\mathbf{1 7}$ & $\mathrm{CH}_{3}$ & $\mathrm{CH}_{3}$ & $70.71 / 70.73$ & $6.10 / 6.07$ & $13.56 / 13.57$ \\
$\mathbf{1 8}$ & $\mathrm{CH}_{3}$ & $\mathrm{~F}$ & $74.21 / 74.19$ & $4.87 / 4.91$ & $14.56 / 14.51$ \\
$\mathbf{1 9}$ & $\mathrm{CH}_{3}$ & $\mathrm{NO}_{2}$ & $71.91 / 71.94$ & $6.21 / 6.19$ & $13.54 / 13.51$ \\
$\mathbf{2 0}$ & $\mathrm{C}_{2} \mathrm{H}_{5}$ & $\mathrm{H}$ & $73.50 / 73.49$ & $5.92 / 5.94$ & $12.82 / 12.83$ \\
$\mathbf{2 1}$ & $\mathrm{C}_{2} \mathrm{H}_{5}$ & $\mathrm{Cl}$ & $70.95 / 70.94$ & $6.84 / 6.82$ & $13.12 / 13.11$ \\
$\mathbf{2 2}$ & $\mathrm{C}_{2} \mathrm{H}_{5}$ & $\mathrm{Br}$ & $74.51 / 74.49$ & $5.81 / 5.82$ & $14.12 / 14.14$ \\
$\mathbf{2 3}$ & $\mathrm{C}_{2} \mathrm{H}_{5}$ & $\mathrm{CH}$ & $72.71 / 72.73$ & $4.87 / 4.84$ & $12.54 / 12.53$ \\
$\mathbf{2 4}$ & $\mathrm{C}_{2} \mathrm{H}_{5}$ & $\mathrm{~F}$ & $74.31 / 74.32$ & $6.47 / 6.43$ & $13.01 / 13.02$ \\
$\mathbf{2 5}$ & $\mathrm{C}_{2} \mathrm{H}_{5}$ & $\mathrm{NO}_{2}$ & $70.61 / 70.62$ & $4.32 / 4.31$ & $14.64 / 14.65$ \\
\hline
\end{tabular}

Synthesis

From the literature precedent ${ }^{24}$ 3-alkyl 2,6-diarylpiperidin-4-one (1-14) were prepared (Scheme 1) by the condensation of appropriate ketones, aldehydes and ammonium acetate in $1: 2: 1$ ratio.

General procedure for the synthesis of 3-alkyl-2,6-diarylpiperidin-4- one-2-thienoyl hydrazone (14-25)

A mixture of 3-alkyl-2,6-diarylpiperidin-4-one (1-14) (1mmol), 2-thiophene-carboxylic acid hydrazide $(1.5 \mathrm{mmol})$ in methanol and chloroform $(1: 1 \mathrm{v} / \mathrm{v})$ and a few drops of acetic acid was added and refluxed for 2-4 hours. On completion of the reaction time, a solid mass was formed, which was then cooled to room temperature. The precipitate was filtered off and washed with ice-cooled water-ethanol mixture. The crude product was recrystallized from ethanol. 


\section{Spectral Data}

\section{3-Methyl-2,6-diphenylpiperidin-4-one-2-thienoyl hydrazone (14)}

IR $(\mathrm{KBr})\left(\mathrm{cm}^{-1}\right): 3074,2880(\mathrm{C}-\mathrm{H}$ stretching $), 1650(\mathrm{~N}-\mathrm{C}=\mathrm{O}$ stretching $) .{ }^{1} \mathrm{H}$ NMR $(\delta \mathrm{ppm})$ : $1.09\left(\mathrm{~d}, 3 \mathrm{H}, \mathrm{CH}_{3}\right.$ at $\left.\mathrm{C}-3\right), 2.07(\mathrm{~s}, 1 \mathrm{H}, \mathrm{N}-\mathrm{H}$ at piperidine ring $), 2.68\left(\mathrm{t}, 1 \mathrm{H}, \mathrm{H}_{3 \mathrm{e}}\right), 2.25(\mathrm{t}, 1 \mathrm{H}$, $\left.\mathrm{H}_{5 \mathrm{e}}\right), 3.36\left(\mathrm{~d}, 1 \mathrm{H}, \mathrm{H}_{5 \mathrm{e}}\right), 3.59\left(\mathrm{~d}, 1 \mathrm{H}, \mathrm{H}_{2 \mathrm{a}}\right), 3.93\left(\mathrm{~d}, 1 \mathrm{H}, \mathrm{H}_{6 \mathrm{a}}\right), 9.90(\mathrm{~s}, 1 \mathrm{H}, \mathrm{HN}-\mathrm{C}=\mathrm{O}), 7.06-$ 7.96 (m, $14 \mathrm{H}$, aryl and thienoyl ring protons). ${ }^{13} \mathrm{C}$ NMR $(\delta \mathrm{ppm}): 13.52\left(\mathrm{CH}_{3}\right), 35.79(\mathrm{C}-5)$, 46.04(C-3), 60.91(C-6), 69.44(C-2), 163.17(HN-C=O), 142.59 and 143.14(ipso carbons), 126.39-135.54 and 156.46(aryl and thienoyl ring carbons).

\section{3-Methyl-2,6-bis(p-chlorophenyl)piperidin-4-one-2-thienoyl hydrazone (15)}

IR $(\mathrm{KBr})\left(\mathrm{cm}^{-1}\right)$ : 3070, 2878(C-H stretching), 1648( $\mathrm{N}-\mathrm{C}=\mathrm{O}$ stretching). ${ }^{1} \mathrm{H}$ NMR $(\delta \mathrm{ppm})$ : 0.94(d, $3 \mathrm{H}, \mathrm{CH}_{3}$ at $\left.\mathrm{C}-3\right), 2.02(\mathrm{~s}, 1 \mathrm{H}, \mathrm{N}-\mathrm{H}$ at piperidine ring $), 2.56\left(\mathrm{t}, 1 \mathrm{H}, \mathrm{H}_{3 \mathrm{e}}\right), 2.08(\mathrm{~d}, 1 \mathrm{H}$, $\left.\mathrm{H}_{5 \mathrm{e}}\right), 2.95\left(\mathrm{~s}, 1 \mathrm{H}, \mathrm{H}_{5 \mathrm{e}}\right), 3.51\left(\mathrm{~d}, 1 \mathrm{H}, \mathrm{H}_{2 \mathrm{a}}\right), 3.83\left(\mathrm{~d}, 1 \mathrm{H}, \mathrm{H}_{6 \mathrm{a}}\right), 10.93(\mathrm{~s}, 1 \mathrm{H}, \mathrm{HN}-\mathrm{C}=\mathrm{O}), 7.17-$ 8.03 $(\mathrm{m}, 12 \mathrm{H}$, aryl and thienoyl ring protons $),{ }^{13} \mathrm{C} \mathrm{NMR}(\mathrm{sppm}): 13.43\left(\mathrm{CH}_{3}\right), 30.66(\mathrm{C}-5)$, 45.02 (C-3), 59.01(C-6), 67.73(C-2), 162.02(HN-C=O), 142.03, 142.87(ipso carbons), 127.99-134.35 and 153.84(aryl and thienoyl ring carbons).

\section{3-Methyl-2,6-bis(p-bromophenyl)piperidin-4-one-2-thienoyl hydrazone (16)}

IR $(\mathrm{KBr})\left(\mathrm{cm}^{-1}\right)$ : 3072, 2884(C-H stretching), 1644(N-C=O stretching). ${ }^{1} \mathrm{H}$ NMR $(\delta p p m)$ : $0.93\left(\mathrm{~s}, 3 \mathrm{H}, \mathrm{CH}_{3}\right.$ at $\left.\mathrm{C}-3\right), 2.08\left(\mathrm{~m}, 2 \mathrm{H}, \mathrm{N}-\mathrm{H}\right.$ at piperidine ring, $\left.\mathrm{H}_{5 \mathrm{e}}\right), 2.53\left(\mathrm{t}, 1 \mathrm{H}, \mathrm{H}_{3 \mathrm{e}}\right), 2.93$ $\left(\mathrm{s}, 1 \mathrm{H}, \mathrm{H}_{5 \mathrm{a}}\right), 3.44\left(\mathrm{~s}, 1 \mathrm{H}, \mathrm{H}_{2 \mathrm{a}}\right), 3.81\left(\mathrm{~d}, 1 \mathrm{H}, \mathrm{H}_{6 \mathrm{a}}\right), 10.91(\mathrm{~s}, 1 \mathrm{H}, \mathrm{HN}-\mathrm{C}=\mathrm{O}), 7.16-8.02(\mathrm{~m}, 12 \mathrm{H}$,

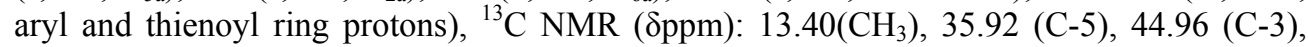
$59.06(\mathrm{C}-6), 67.76(\mathrm{C}-2), 162.05(\mathrm{HN}-\mathrm{C}=\mathrm{O}), 142.42$ and 143.27(ipso carbons), $119.98-$ 134.42 and 156.88 (aryl and thienoyl ring carbons).

\section{3-Methyl-2,6-bis(p-methylphenyl)piperidin-4-one-2-thienoyl hydrazone (17)}

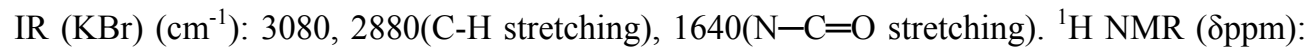
1.08(s, $3 \mathrm{H}, \mathrm{CH}_{3}$ at $\left.\mathrm{C}-3\right), 2.02\left(\mathrm{~s}, 1 \mathrm{H}, \mathrm{N}-\mathrm{H}\right.$ at piperidine ring), 2.07(t, $\left.1 \mathrm{H}, \mathrm{H}_{5 \mathrm{e}}\right), 2.34(\mathrm{~s}, 6 \mathrm{H}$, $\mathrm{CH}_{3}$ at aryl ring), 2.62(d, 1H, $\left.\mathrm{H}_{3 \mathrm{e}}\right), 3.03\left(\mathrm{~d}, 1 \mathrm{H}, \mathrm{H}_{5 \mathrm{a}}\right), 3.54\left(\mathrm{~s}, 1 \mathrm{H}, \mathrm{H}_{2 \mathrm{a}}\right), 3.86\left(\mathrm{~d}, 1 \mathrm{H}, \mathrm{H}_{6 \mathrm{a}}\right)$, 9.07(s, 1H, HN-C=O), 7.09-8.09(m, 12H, aryl and thienoyl ring protons), ${ }^{13} \mathrm{C}$ NMR

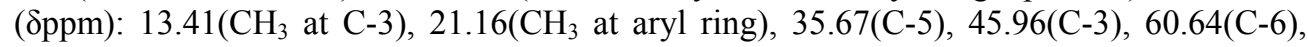
69.11 (C-2), 162.72( $\mathrm{HN}-\mathrm{C}=\mathrm{O}$ ), 140.10 and 139.60(ipso carbons), 126.52-137.59 and 156.23(aryl and thienoyl ring carbons).

\section{3-Methyl-2,6-bis(p-fluorophenyl)piperidin-4-one-2-thienoyl hydrazone (18)}

IR $(\mathrm{KBr})\left(\mathrm{cm}^{-1}\right)$ : 3080, 2920(C-H stretching), 1640(N-C=O stretching). ${ }^{1} \mathrm{H}$ NMR $(\delta \mathrm{ppm})$ : 1.07( $\mathrm{d}, 3 \mathrm{H}, \mathrm{CH}_{3}$ at piperidine ring), $2.02\left(\mathrm{~s}, 1 \mathrm{H}, \mathrm{N}-\mathrm{H}\right.$ at piperidin ring), $2.19\left(\mathrm{t}, 1 \mathrm{H}, \mathrm{H}_{5 \mathrm{e}}\right), 2.61$ $\left(\mathrm{s}, 1 \mathrm{H}, \mathrm{H}_{3 \mathrm{e}}\right), 3.23\left(\mathrm{~d}, 1 \mathrm{H}, \mathrm{H}_{5 \mathrm{a}}\right), 3.58\left(\mathrm{~d}, 1 \mathrm{H}, \mathrm{H}_{2 \mathrm{a}}\right), 3.91\left(\mathrm{~d}, 1 \mathrm{H}, \mathrm{H}_{6 \mathrm{a}}\right), 9.71(\mathrm{~s}, 1 \mathrm{H}, \mathrm{HN}-\mathrm{C}=\mathrm{O})$, $7.04-7.95(\mathrm{~m}, 12 \mathrm{H}$, aryl and thienoyl ring protons $),{ }^{13} \mathrm{C} \mathrm{NMR}(\delta \mathrm{ppm}): 13.36\left(\mathrm{CH}_{3}\right.$ at piperidine ring), 35.87(C-5), 46.12(C-3), 60.19(C-6), 68.59(C-2), 163.64( $\mathrm{HN}-\mathrm{C}=\mathrm{O}), 138.82$ and 138.24(ipso carbons), 115.26-135.49 and 155.65(aryl and thienoyl ring carbons).

\section{3-Methyl-2,6-bis(p-nitrophenyl)piperidin-4-one-2-thienoyl hydrazone (19)}

IR (KBr) $\left(\mathrm{cm}^{-1}\right)$ : 3072, 2913(C-H stretching), 1632(N-C=O stretching). ${ }^{1} \mathrm{H}$ NMR( $\left.\delta p p m\right)$ : $1.12\left(\mathrm{~d}, 3 \mathrm{H}, \mathrm{CH}_{3}\right.$ at piperidine ring), 2.12(s, $1 \mathrm{H}, \mathrm{N}-\mathrm{H}$ at piperidine ring $), 2.20\left(\mathrm{t}, 1 \mathrm{H}, \mathrm{H}_{5 \mathrm{e}}\right), 2.72$ $\left(\mathrm{s}, 1 \mathrm{H}, \mathrm{H}_{3 \mathrm{a}}\right), 3.32\left(\mathrm{~d}, 1 \mathrm{H}, \mathrm{H}_{5 \mathrm{a}}\right), 3.61\left(\mathrm{~d}, 1 \mathrm{H}, \mathrm{H}_{2 \mathrm{a}}\right), 3.94\left(\mathrm{~d}, 1 \mathrm{H}, \mathrm{H}_{6 \mathrm{a}}\right), 9.84(\mathrm{~s}, 1 \mathrm{H}, \mathrm{HN}-\mathrm{C}=\mathrm{O}), 7.11-$ $7.84\left(\mathrm{~m}, 10 \mathrm{H}\right.$, aryl and thienoyl ring protons), ${ }^{13} \mathrm{C} \mathrm{NMR}(\mathrm{\delta ppm}): 13.20\left(\mathrm{CH}_{3}\right.$ at piperidine ring), 34.24(C-5), 45.72(C-3), 61.21(C-6), 67.84(C-2),162.24( $\mathrm{HN}-\mathrm{C}=\mathrm{O}), 140.20$ and 139.82 (ipso carbons), 118.92-135.21 and 154.46 (aryl and thienoyl ring carbons). 


\section{3-Ethyl-2,6-diphenylpipseridin-4-one-2-thienoyl hydrazone (20)}

IR $(\mathrm{KBr})\left(\mathrm{cm}^{-1}\right): 3070,2888(\mathrm{C}-\mathrm{H}$ stretching $), 1646\left(\mathrm{~N}-\mathrm{C}=\mathrm{O}\right.$ stretching). ${ }^{1} \mathrm{H}$ NMR $(\delta \mathrm{ppm})$ : $0.08\left(\mathrm{t}, 3 \mathrm{H}, \mathrm{CH}_{2}-\mathrm{CH}_{3}\right.$ at $\left.\mathrm{C}-3\right), 1.41\left(\mathrm{t}, 2 \mathrm{H}, \mathrm{CH}_{2}-\mathrm{CH}_{3}\right), 2.01(\mathrm{~s}, 1 \mathrm{H}, \mathrm{N}-\mathrm{H}$ at piperidine ring), 2.24(t, $\left.1 \mathrm{H}, \mathrm{H}_{5 \mathrm{e}}\right), 2.54\left(\mathrm{t}, 1 \mathrm{H}, \mathrm{H}_{3 \mathrm{e}}\right), 3.22\left(\mathrm{~d}, 1 \mathrm{H}, \mathrm{H}_{5 \mathrm{a}}\right), 3.73\left(\mathrm{~d}, 1 \mathrm{H}, \mathrm{H}_{2 \mathrm{a}}\right), 3.93\left(\mathrm{~d}, 1 \mathrm{H}, \mathrm{H}_{6 \mathrm{a}}\right), 9.57(\mathrm{~s}, 1 \mathrm{H}$, $\mathrm{HN}-\mathrm{C}=\mathrm{O}), 7.06-7.99(\mathrm{~m}, 12 \mathrm{H}$, aryl and thienoyl ring protons $){ }^{13} \mathrm{C} \mathrm{NMR}(\delta \mathrm{ppm}): 12.48\left(\mathrm{CH}_{2}-\right.$ $\left.\underline{\mathrm{CH}}_{3}\right), 19.18\left(\mathrm{CH}_{2}-\mathrm{CH}_{3}\right), 36.17(\mathrm{C}-5), 52.58(\mathrm{C}-3), 60.96(\mathrm{C}-6), 67.78(\mathrm{C}-2), 162.97(\mathrm{HN}-\mathrm{C}=\mathrm{O})$, 142.54 and 143.10 (ipso carbons), $126.45-135.45$ and 154.85 (aryl and thienoyl ring carbons).

\section{3-Ethyl-2,6-bis(p-chlorophenyl)piperidin-4-one-2-thienoyl hydrazone (21)}

IR $(\mathrm{KBr})\left(\mathrm{cm}^{-1}\right)$ : 3074, 2920(C-H stretching), 1640(N-C=O stretching). ${ }^{1} \mathrm{H}$ NMR $(\delta p p m): 1.37(\mathrm{t}$, $3 \mathrm{H}, \mathrm{CH}_{2}-\mathrm{CH}_{3}$ at piperidine ring ), 1.09 and $1.84\left(\mathrm{~m}, 2 \mathrm{H}, \mathrm{CH}_{2}-\mathrm{CH}_{3}\right), 1.96(\mathrm{~s}, 1 \mathrm{H}, \mathrm{N}-\mathrm{H}$ at piperidine ring), $2.46\left(\mathrm{t}, 1 \mathrm{H}, \mathrm{H}_{3 \mathrm{e}}\right), 3.21\left(\mathrm{~d}, 1 \mathrm{H}, \mathrm{H}_{5 \mathrm{a}}\right), 3.70\left(\mathrm{~d}, 1 \mathrm{H}, \mathrm{H}_{2 \mathrm{a}}\right), 3.90\left(\mathrm{~d}, 1 \mathrm{H}, \mathrm{H}_{6 \mathrm{a}}\right), 9.63(\mathrm{~s}, 1 \mathrm{H}$, $\mathrm{HN}-\mathrm{C}=\mathrm{O}), 7.08-7.93\left(\mathrm{~m}, 10 \mathrm{H}\right.$, aryl and thienoyl ring protons), ${ }^{13} \mathrm{C} \mathrm{NMR}(\delta \mathrm{ppm}): 12.45\left(\mathrm{CH}_{2}-\right.$ $\left.\underline{\mathrm{CH}}_{3}\right), 19.08\left(\mathrm{CH}_{2}-\mathrm{CH}_{3}\right), 30.97(\mathrm{C}-5), 52.56(\mathrm{C}-3), 60.25(\mathrm{C}-6), 66.96(\mathrm{C}-2), \quad 161.54(\mathrm{HN}-\mathrm{C}=\mathrm{O})$, 141.45 and 140.86(ipso carbons), 126.51-134.22 and 153.79(aryl and thienoyl ring carbons).

\section{3-Ethyl-2,6 -bis(p-bromophenyl)piperidin-4-one-2-thienoyl hydrazone (22)}

IR $(\mathrm{KBr})\left(\mathrm{cm}^{-1}\right): 3071,2886(\mathrm{C}-\mathrm{H}$ stretching $), 1642\left(\mathrm{~N}-\mathrm{C}=\mathrm{O}\right.$ stretching). ${ }^{1} \mathrm{H}$ NMR $(\delta \mathrm{ppm})$ : $0.78\left(\mathrm{t}, 3 \mathrm{H}, \mathrm{CH}_{2}-\mathrm{CH}_{3}\right.$ at $\left.\mathrm{C}-3\right), 1.21\left(\mathrm{~s}, 2 \mathrm{H}, \mathrm{CH}_{2}-\mathrm{CH}_{3}\right), 1.79\left(\mathrm{~s}, 1 \mathrm{H}, \mathrm{CH}_{2}-\mathrm{CH}_{3}\right), 2.03(\mathrm{~s}, 1 \mathrm{H}$, $\mathrm{N}-\mathrm{H}$ at piperidine ring), $2.50\left(\mathrm{t}, 1 \mathrm{H}, \mathrm{H}_{3 \mathrm{e}}\right), 2.88\left(\mathrm{~s}, 1 \mathrm{H}, \mathrm{H}_{5 \mathrm{e}}\right), 3.63\left(\mathrm{~d}, 1 \mathrm{H}, \mathrm{H}_{2 \mathrm{e}}\right), 3.84(\mathrm{~d}, 1 \mathrm{H}$, $\left.\mathrm{H}_{6 \mathrm{a}}\right), 10.88(\mathrm{~s}, 1 \mathrm{H}, \mathrm{HN}-\mathrm{C}=\mathrm{O}), 7.16-8.03(\mathrm{~m}, 10 \mathrm{H}$, aryl and thienoyl ring protons $),{ }^{13} \mathrm{C}$

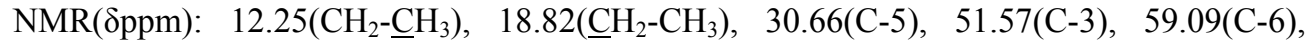
66.22(C-2), 167.44(HN- $\mathrm{C}=\mathrm{O}), 143.26$ and 142.38(ipso carbons), 119.98-134.30 and 155.59(aryl and thienoyl ring carbons).

\section{3-Ethyl-2,6-bis(p-methylphenyl)piperidin-4-one-2-thienoyl hydrazone (23)}

IR $(\mathrm{KBr})\left(\mathrm{cm}^{-1}\right)$ : 3074, 2924(C-H stretching), 1640(N-C=O stretching). ${ }^{1} \mathrm{H}$ NMR $(\delta \mathrm{ppm})$ : $0.88\left(\mathrm{t}, 3 \mathrm{H}, \mathrm{CH}_{2}-\underline{\mathrm{C}} \mathrm{H}_{3}\right.$ at piperidine ring ), 1.38 and $1.85\left(\mathrm{~m}, 2 \mathrm{H}, \underline{\mathrm{CH}}_{2}-\mathrm{CH}_{3}\right), 1.95(\mathrm{~s}, 1 \mathrm{H}, \mathrm{N}-\mathrm{H}$ at piperidine ring), 2.20( $\left.\mathrm{t}, 1 \mathrm{H}, \mathrm{H}_{5 \mathrm{e}}\right), 2.51\left(\mathrm{t}, 1 \mathrm{H}, \mathrm{H}_{3 \mathrm{e}}\right), 3.0\left(\mathrm{~d}, 1 \mathrm{H}, \mathrm{H}_{5 \mathrm{a}}\right), 3.68\left(\mathrm{~d}, 1 \mathrm{H}, \mathrm{H}_{2 \mathrm{a}}\right)$, $3.86\left(\mathrm{~d}, 1 \mathrm{H}, \mathrm{H}_{6 \mathrm{a}}\right), 9.17(\mathrm{~s}, 1 \mathrm{H}, \mathrm{HN}-\mathrm{C}=\mathrm{O}), 7.08-8.07(\mathrm{~m}, 10 \mathrm{H}$, aryl and thienoyl ring protons), 2.34(s, $6 \mathrm{H}, \mathrm{CH}_{3}$ at phenyl ring), ${ }^{13} \mathrm{C} \mathrm{NMR}(\delta \mathrm{ppm}): 12.47\left(\mathrm{CH}_{2}-\mathrm{CH}_{3}\right), 19.17\left(\mathrm{CH}_{2}-\mathrm{CH}_{3}\right)$, $21.17\left(\mathrm{CH}_{3}\right.$ at phenyl ring), 36.12(C-5), 52.53(C-3), 60.67(C-6), 67.46(C-2), 164.08 $(\mathrm{HN}-\mathrm{C}=\mathrm{O}), 140.12,139.57,137.56$ and 137.53(ipso carbons), 126.53-135.44 and 154.76(aryl and thienoyl ring carbons).

\section{3-Ethyl-2,6-bis(p-fluorophenyl)piperidin-4-one-2-thienoyl hydrazone (24)}

IR $(\mathrm{KBr})\left(\mathrm{cm}^{-1}\right)$ : 3068, 2918(C-H stretching), 1634(N-C=O stretching). ${ }^{1} \mathrm{H}$ NMR ( $\left.\delta \mathrm{ppm}\right)$ : $0.87\left(\mathrm{t}, 3 \mathrm{H}, \mathrm{CH}_{2}-\underline{\mathrm{CH}}_{3}\right), 1.35$ and $1.86\left(\mathrm{~m}, 2 \mathrm{H}, \underline{\mathrm{CH}}_{2}-\mathrm{CH}_{3}\right), 1.96(\mathrm{~s}, 1 \mathrm{H}, \mathrm{N}-\mathrm{H}$ at piperidine ring), 2.18(t, 1H, $\left.\mathrm{H}_{3 \mathrm{e}}\right), 2.46\left(\mathrm{t}, 1 \mathrm{H}, \mathrm{H}_{5 \mathrm{e}}\right), 3.05\left(\mathrm{~d}, 1 \mathrm{H}, \mathrm{H}_{5 \mathrm{a}}\right), 3.70\left(\mathrm{~d}, 1 \mathrm{H}, \mathrm{H}_{2 \mathrm{a}}\right), 3.89\left(\mathrm{~d}, 1 \mathrm{H}, \mathrm{H}_{6 \mathrm{a}}\right)$, $9.09(\mathrm{~s}, 1 \mathrm{H}, \mathrm{HN}-\mathrm{C}=\mathrm{O}), 7.02-8.08(\mathrm{~m}, 10 \mathrm{H}$, aryl and thienoyl ring protons $),{ }^{13} \mathrm{C} \mathrm{NMR}$

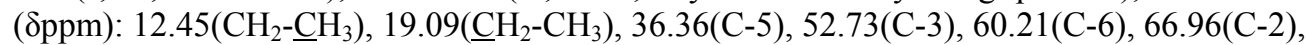
$163.11(\mathrm{HN}-\mathrm{C}=\mathrm{O}), 138.88$ and 138.25 (ipso carbons), 115.30-135.43(aryl and thienoyl ring carbons).

\section{3-Ethyl-2,6-bis(p-nitrophenyl)piperidin-4-one-2-thienoyl hydrazone (25)}

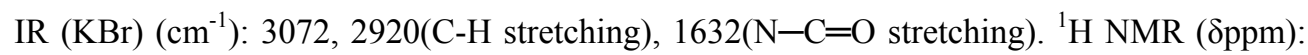
$1.10\left(\mathrm{t}, 3 \mathrm{H}, \mathrm{CH}_{2}-\mathrm{CH}_{3}\right.$ at piperidine ring), 1.34 and $1.80\left(\mathrm{~m}, 2 \mathrm{H}, \underline{\mathrm{CH}}_{2}-\mathrm{CH}_{3}\right), 1.92(\mathrm{~s}, 1 \mathrm{H}, \mathrm{N}-\mathrm{H}$ at piperidine ring $), 2.14\left(\mathrm{t}, 1 \mathrm{H}, \mathrm{H}_{5 \mathrm{e}}\right), 2.47\left(\mathrm{t}, 1 \mathrm{H}, \mathrm{H}_{3 \mathrm{e}}\right), 3.12\left(\mathrm{~d}, 1 \mathrm{H}, \mathrm{H}_{5 \mathrm{a}}\right), 3.64\left(\mathrm{~d}, 1 \mathrm{H}, \mathrm{H}_{2 \mathrm{a}}\right)$, 
3.92(d, $\left.1 \mathrm{H}, \mathrm{H}_{6 \mathrm{a}}\right), 9.82(\mathrm{~s}, 1 \mathrm{H}, \mathrm{HN}-\mathrm{C}=\mathrm{O}), 7.11-8.12(\mathrm{~m}, 10 \mathrm{H}$, aryl and thienoyl ring protons),

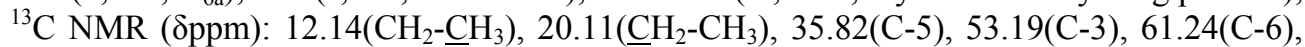
68.11(C-2), 163.21(HN- $\mathrm{C}=\mathrm{O}), 141.23,140.24,139.24$ and 139.12 (ipso carbons), $125.87-$ 135.42 and 151.24(aryl and thienoyl ring carbons).

\section{Antimicrobial activity (Structure-activity relationship)}

The new class of compounds in this paper have been preliminarily evaluated for their antibacterial and antifungal activities against a band of certain selected bacterial (Escherichia coli, Salmonella typhi, Staphylococcus aureus, Klebsiella pneumonia and Pseudomonas aeruginosa) and fungal (Aspergillus niger, Aspergillus flavus, Candida albicans and Rhizopus $s p$.,) pathogens in vitro by conventional twofold serial dilution method ${ }^{25}$. The obtained results were compared to ciprofloxacin and Amphotericin B which were used as the standard drugs for bacterial and fungal strains respectively. The antibacterial and antifungal potencies of the synthesized compounds are reproduced in Table 2 and 3 respectively.

Table 2. In vitro antibacterial activities of compounds (14-25) against selected bacterial strain in $\mu \mathrm{g} / \mathrm{mL}$

\begin{tabular}{cccccccc}
\hline Compds & $\mathrm{R}_{1}$ & $\mathrm{X}$ & E.Coli & $\begin{array}{c}\text { Salmonella } \\
\text { typhi }\end{array}$ & $\begin{array}{c}\text { Staphylococcus } \\
\text { aureus }\end{array}$ & $\begin{array}{c}\text { Klebsiella } \\
\text { pneumonia }\end{array}$ & $\begin{array}{c}\text { Pseudomona } \\
\text { aeruginosa }\end{array}$ \\
\hline $\mathbf{1 4}$ & $\mathrm{CH}_{3}$ & $\mathrm{H}$ & 200 & 100 & - & 200 & 100 \\
$\mathbf{1 5}$ & $\mathrm{CH}_{3}$ & $\mathrm{Cl}$ & 25 & 25 & 12.5 & 25 & 25 \\
$\mathbf{1 6}$ & $\mathrm{CH}_{3}$ & $\mathrm{Br}$ & 12.5 & 25 & 50 & 25 & 25 \\
$\mathbf{1 7}$ & $\mathrm{CH}_{3}$ & $\mathrm{CH}_{3}$ & 100 & 200 & 100 & 50 & 100 \\
$\mathbf{1 8}$ & $\mathrm{CH}_{3}$ & $\mathrm{~F}$ & 12.5 & 12.5 & 25 & 12.5 & 25 \\
$\mathbf{1 9}$ & $\mathrm{CH}_{3}$ & $\mathrm{NO}_{2}$ & 50 & 100 & 50 & 25 & 100 \\
$\mathbf{2 0}$ & $\mathrm{C}_{2} \mathrm{H}_{5}$ & $\mathrm{H}$ & 200 & 100 & 100 & 200 & 100 \\
$\mathbf{2 1}$ & $\mathrm{C}_{2} \mathrm{H}_{5}$ & $\mathrm{Cl}$ & 25 & 25 & 12.5 & 25 & 25 \\
$\mathbf{2 2}$ & $\mathrm{C}_{2} \mathrm{H}_{5}$ & $\mathrm{Br}$ & 25 & 12.5 & 12.5 & 25 & 25 \\
$\mathbf{2 3}$ & $\mathrm{C}_{2} \mathrm{H}_{5}$ & $\mathrm{CH}$ & 100 & 100 & 200 & 100 & 100 \\
$\mathbf{2 4}$ & $\mathrm{C}_{2} \mathrm{H}_{5}$ & $\mathrm{~F}$ & 12.5 & 25 & 12.5 & 25 & 12.5 \\
$\mathbf{2 5}$ & $\mathrm{C}_{2} \mathrm{H}_{5}$ & $\mathrm{NO}_{2}$ & 50 & 50 & 100 & 50 & 50 \\
Ciprofloacin & & 25 & 50 & 12.5 & 12.5 & 25 \\
\hline
\end{tabular}

Table 3. In vitro antifungal activities of compounds (14-25)

\begin{tabular}{ccccccc}
\hline Compounds & $\mathrm{R}_{1}$ & $\mathrm{X}$ & A.niger & A. flavus & Candida albicans & Rhizopus \\
\hline $\mathbf{1 4}$ & $\mathrm{CH}_{3}$ & $\mathrm{H}$ & - & 200 & - & 200 \\
$\mathbf{1 5}$ & $\mathrm{CH}_{3}$ & $\mathrm{Cl}$ & 25 & 12.5 & 25 & 25 \\
$\mathbf{1 6}$ & $\mathrm{CH}_{3}$ & $\mathrm{Br}$ & 12.5 & 25 & 25 & 25 \\
$\mathbf{1 7}$ & $\mathrm{CH}_{3}$ & $\mathrm{CH}_{3}$ & 100 & 50 & 100 & 100 \\
$\mathbf{1 8}$ & $\mathrm{CH}_{3}$ & $\mathrm{~F}$ & 12.5 & 12.5 & 12.5 & 25 \\
$\mathbf{1 9}$ & $\mathrm{CH}_{3}$ & $\mathrm{NO}_{2}$ & 50 & 100 & 100 & 50 \\
$\mathbf{2 0}$ & $\mathrm{C}_{2} \mathrm{H}_{5}$ & $\mathrm{H}$ & 200 & 100 & - & 200 \\
$\mathbf{2 1}$ & $\mathrm{C}_{2} \mathrm{H}_{5}$ & $\mathrm{Cl}$ & 25 & 12.5 & 25 & 12.5 \\
$\mathbf{2 2}$ & $\mathrm{C}_{2} \mathrm{H}_{5}$ & $\mathrm{Br}$ & 25 & 12.5 & 25 & 25 \\
$\mathbf{2 3}$ & $\mathrm{C}_{2} \mathrm{H}_{5}$ & $\mathrm{CH}$ & 100 & 50 & 50 & 50 \\
$\mathbf{2 4}$ & $\mathrm{C}_{2} \mathrm{H}_{5}$ & $\mathrm{~F}$ & 12.5 & 12.5 & 25 & 12.5 \\
$\mathbf{2 5}$ & $\mathrm{C}_{2} \mathrm{H}_{5}$ & $\mathrm{NO}_{2}$ & 100 & 50 & 50 & 100 \\
Amphotericin B & & 25 & 25 & 25 & 25 \\
\hline
\end{tabular}


Compounds 14 and 20 without any substituent at para position of the aryl moieties at C-2 and C-6 position of the piperidine exhibited antibacterial activity in vitro at $200 \mu \mathrm{g} / \mathrm{mL}$ against all the tested organism except Salmonella typhi and P.aeruginosa. They inhibit both the organism at MIC of $100 \mu \mathrm{g} / \mathrm{mL}$.

Introduction of chloro, bromo and fluoro groups at the para position of the aryl moieties at $\mathrm{C}-2$ and $\mathrm{C}-6$ in piperidine in compound 15, 16 and 18 produced twice the activity against all the organisms. Replacement of halo groups present at the para position of the aryl moieties at C-2 and C-6 of $\mathbf{1 4}$ by nitro group yielded moderate activity against all the tested organism except Salmonella typhi and P.aeruginosa.

Introduction of an ethyl groups in place of methyl function in 14 (compound 20) exhibited appreciable inhibition potency towards the tested bacteria strains in the range of $100-200 \mu \mathrm{g} / \mathrm{mL}$. Introduction of chloro, bromo and fluoro groups at the para position of the aryl moieties at C-2 and C-6 in piperidine in compound 21, 22 and 24 produced antibacterial activity in the range of $12.5-25 \mu \mathrm{g} / \mathrm{mL}$.

Replacement of halo moieties present at the para position of aryl groups by nitro group (compound 25) produced antibacterial activity in the range of $50-100 \mu \mathrm{g} / \mathrm{mL}$. Compound 23, which have methyl group at the para position C-2 and C-6 in piperidine ring exhibited antibacterial activity in the region of $100-200 \mu \mathrm{g} / \mathrm{mL}$.

\section{Antifungal activities}

The in vitro antifungal activity of the novel compounds 14-25 were examined against the four fungal strains Aspergillus niger, Aspergillus flavus, Candida albicans and Rhizopus sp., Here, Amphotericin B was used as standard drug.

The compound $\mathbf{1 4}$ and $\mathbf{2 0}$ without any substituents at the para position of phenyl groups present at the carbon atom adjacent to the heterocyclic nitrogen exhibited antifungal activity in the region 100-200 $\mu \mathrm{g} / \mathrm{mL}$. However, compound 14 against A.niger and 20 against Candida albicans did not show antifungal activity even at a maximum concentration of 200 $\mu \mathrm{g} / \mathrm{mL}$. Compounds 15, 16, 18, 21, 22 and 24, which have electron withdrawing chloro, bromo and fluoro substitution at the para position of phenyl rings attached to C-2 and C-6 carbons of piperdine moiety exerted activity in the region of $12.5-25 \mu \mathrm{g} / \mathrm{mL}$.

Due to introduction of a methyl group in place of the halo function present at the para position of the phenyl groups (compound 17 and 23), the activity was suppressed against all the tested organisms. Introductions of nitro group at the para position of phenyl rings attached to C-2 and C-6 carbons of piperidine moiety shows antifungal activities in the range of $50-100 \mu \mathrm{g} / \mathrm{mL}$.

\section{Results and Discussion}

The obvious synthetic pathway that leads to the title compounds is represented in Scheme 1 structures of all the synthesized compounds were established on the basis of ${ }^{1} \mathrm{H}$ NMR and ${ }^{13} \mathrm{C}$ NMR spectral data. The purities of the compounds were checked by elemental analysis. The analytic data agreed well with their proposed molecular formulas. ${ }^{1} \mathrm{H}$ NMR assignment of compounds 14-25 is made based on their one- and two- dimensional NMR spectral studies. In the ${ }^{1} \mathrm{H}$ NMR spectra of the target hydrazones, a broad and more downfield $\mathrm{D}_{2} \mathrm{O}$ exchangeable singlet at $9.73 \mathrm{ppm}$ was characteristic of the $\mathrm{N}-\mathrm{H}$ amide group. The two doublet signal appeared at 3.94 and $3.59 \mathrm{ppm}$, corresponding to one proton integrals. These two signals are attributed to benzylic protons $\mathrm{H}_{6 \mathrm{a}}$ and $\mathrm{H}_{2 \mathrm{a}}$ respectively. However, signals that appeared at 3.31 and $2.25 \mathrm{ppm}$ should be due to the $\mathrm{H}_{5 \mathrm{a}}$ and $\mathrm{H}_{5 \mathrm{e}}$. The methyl proton appeared at $1.09 \mathrm{ppm}$ corresponding to three proton integrals. A triplet appeared at $2.66 \mathrm{ppm}$ due to the 
$\mathrm{H}_{3 \mathrm{a}}$, corresponding to one proton integral. Aromatic protons appeared as a multiplet in the region of 7.06-7.96 ppm. In the ${ }^{13} \mathrm{C}$ NMR spectrum of compound 15, an absorptions at $13.52 \mathrm{ppm}$ is assigned to methyl carbon at C-3. The benzylic carbon C-2 and C-6 are observed at 69.44 and $60.91 \mathrm{ppm}$. The ${ }^{13} \mathrm{C}$ resonance at 46.04 and $35.79 \mathrm{ppm}$ is due to $\mathrm{C}-3$ and $\mathrm{C}-5$. The chemical shift value at $163.17 \mathrm{ppm}$ is assigned to $\mathrm{C}=\mathrm{O}$ carbon. The ${ }^{13} \mathrm{C}$ resonance at 143.14 and $142.59 \mathrm{ppm}$ is assigned to ipso carbons of two phenyl substituents at C-2 and C-4. The aromatic carbons and thiophene carbons appeared in the region of 126.37-156.46 ppm.<smiles>CC(=O)C[18O]</smiles><smiles>[X]c1ccc(C=O)cc1</smiles><smiles>[X]c1ccc(C=O)cc1</smiles>

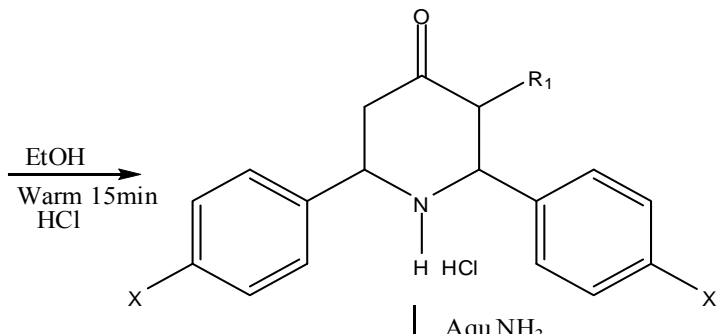

$\mathrm{NH}_{4}^{+}$<smiles>CC(=O)O</smiles>

$\begin{array}{ccc} & & \\ & & \\ & & \\ & & \\ & & \\ \text { Compounds } & \mathrm{R}_{1} & \mathrm{X} \\ 14 & \mathrm{CH}_{3} & \mathrm{H} \\ 15 & \mathrm{CH}_{3} & \mathrm{Cl} \\ 16 & \mathrm{CH}_{3} & \mathrm{Br} \\ 17 & \mathrm{CH}_{3} & \mathrm{CH} \\ 18 & \mathrm{CH}_{3} & \mathrm{~F} \\ 19 & \mathrm{CH}_{3} & \mathrm{NO}_{2} \\ 20 & \mathrm{C}_{2} \mathrm{H}_{5} & \mathrm{H} \\ 21 & \mathrm{C}_{2} \mathrm{H}_{5} & \mathrm{Cl}^{2} \\ 22 & \mathrm{C}_{2} \mathrm{H}_{5} & \mathrm{Br} \\ 23 & \mathrm{C}_{2} \mathrm{H}_{5} & \mathrm{CH}_{3} \\ 24 & \mathrm{C}_{2} \mathrm{H}_{5} & \mathrm{~F} \\ 25 & \mathrm{C}_{2} \mathrm{H}_{5} & \mathrm{NO}_{2}\end{array}$
Ice, $20{ }^{\circ} \mathrm{C}$<smiles>[X]c1ccc(C2CC(=O)C([R1])C(c3ccc([X])cc3)N2CC(C)C(C)(C)C(C)(C)C(=O)O)cc1</smiles><smiles>[X]c1ccc(C2C/C(=N\N([2H])C(=O)c3cccs3)C([R8])C(c3ccc([X])cc3)N2N)cc1</smiles>

$14-25$

\section{Scheme 1}




\section{Conclusion}

A close examination of the in vitro antibacterial and antifungal activity profile of the new 2 , 6-diaryl piperidin-4-one-2-thienoyl hydrazone against all tested bacterial and fungal organism gives a clear picture about the structure-activity correlations among the compound 14-25 under study. Compounds 14-25 with chloro, bromo and fluoro functions at the para position of the aryl groups present at the C-2 and C- 6 position of the piperidine moiety along with methyl and ethyl substituent at the $\mathrm{C}$ - 3 position exerted a strong activity, while the activity was not significant for compounds $\mathbf{1 4}$ and $\mathbf{2 0}$ without any substituent at the para position of phenyl groups.

Similarly, against the tested fungal strains, compounds 15, 16, 18, 21, 22 and 24 with fluoro, bromo and chloro functions at the para position of phenyl rings attached at C-2 and C- 6 carbons of piperidine moiety along with methyl and ethyl substituent at the position C3 of the piperdine ring exerted a good range of biological activities, while the activity was not significant for compounds $\mathbf{1 4}$ and $\mathbf{2 0}$ without any substituents at the para position of the phenyl groups.

Furthermore, the observed marked antifungal and antibacterial activities may be considered as key step for the building of novel chemical entities with comparable pharmacological profile to that of the standard drugs.

\section{Acknowledgement}

We are thankful to chemistry section FEAT, Annamalai University for the facilities provided. We are also thankful to NMR facilities to record NMR spectra Department of Chemistry Annamalai University.

\section{References}

1. EI-Subbagh H I, Abu-Zaid S M, Mahran M A, Badria F A and Al.Obaid A M, J Med Chem., 2000, 43(15), 2915-2921; DOI:10.1021/JM000038M

2. Watson A A, Fleet G W J, Asano N, Molyneux R J and Nugh R J, Phytochemistry, 2001, 56(3), 265-295; DOI:10.1016/S0031-9422(00)00451-9

3. Richards G J, Juan B C, Mario R A, Roldan M and Peinado C R, Fernando Spen., 1979, 47, 168.

4. Jerom B R and Spencer K H, Eur Pat Appl., 1988, (EP277794).

5. Perumal R V, Adirja M and Shanmugapandian P, Indian Drugs, 2001, 38(3), 156.

6. Bochringer C F and Shochne G M B H, Brif Pat Appl., 1961, (BP866488).

7. Ganellin C R and Spickett R G W, J Med Chem., 1965, 8(5), 619-625; DOI:10.1021/JM00329A015

8. Nikolov M, Stefanora D and Chardanov D, Acta Nerv Super., 1974, 16, 264-267.

9. Kathleen B, Jean-peirre C and Andre H, Eur Pat Appl., 1986, (EP169139).

10. Hagenbach R E and Gysin H, Experentia., 1952, 8, 184.

11. Ileana B, Dobre V and Niculescu-Duvaz I, J Prakt Chem., 1985, 327, 667.

12. Mokio I G, Soldatenkov A T, Federov V O, Ageev E A, Segeeva N D, Lin S, Stashenku E E, Prostakov N S and Andreeva E L, Khim Farm Zh., 1989, 23, 421-427.

13. Dimmock J R and Kumar P K, Curr Med Chem., 1997, 4, 1-22.

14. Kubota H, Kakefuda A, Okamoto Y, Fujii M, Yamamoto O, Yamgiwa Y, Orita M,

15. Prostakov N S and Gaivoronskaya L A, Russ Chem Rev., 1978, 47, 447-469.

16. Fang V S, Minkin C and Goldhaber P, Science, 1971, 172, 163-165.

17. Bohm R and Zeiger G, Pharmazie, 1980, 35, 1-4. 
18. Pratt J W, In Ullmann's Encyclopedia of Industrial Chemistry. 2003, 36, Wiley-VCH, Weinheim, pp. 653-662.

19. Friesen L F, Nelson A G and Van Acker R C, Agron J., 2003, 95, 1342-1347.

20. Fischer R, Lui N, Dutzmann S and Haenssler G, Ger Offen Pat., 1998, 19649093; Chem Abstra., 1998, 129. P 24482.

21. Plath P, Eicken K, Rohr W, Zeeh B and Pommer E H, Ger Offen Pat., 1980, 2854598; Chem Abstr., 1980, 94, P15740f.

22. Eltzer R I, Lewis A D, McMillan F H, Genzer J D, Leonord F and King J A, J Am Pharm Assoc., 1953, 42, 594-600.

23. Toshihiko H, Yoshio S, Yoichi H, Etsuro S and Toshihiko I, Jpn Kokai Pat., 1975, 7576069; Chem Abstr.,1975, 84, P31045g.

24. C.R.Noller C R and Balliah V, J Am Chem Soc., 1948, 70(11), 3853-3855; DOI:10.1021/ja01191a092

25. Dhar M H, Dhar M M, Dhawan B N, Mehrotra B N and Ray C, Indian J Exp Biol., 1968, 6, 232-236. 